\title{
Amphipathic Nanodiamond Supraparticles for Anticancer Drug Loading and Delivery
}

Yue Yu, ${ }^{\dagger}$ Xi Yang, ${ }^{\dagger}$ Ming Liu, ${ }^{\star}$ Masahiro Nishikawa, ${ }^{*}$ Takahiro Tei, ${ }^{\S}$ and Eijiro Miyako ${ }^{*}+$

tDepartment of Materials and Chemistry, Nanomaterials Research Institute (NMRI), National Institute of Advanced Industrial Science and Technology (AIST), Central 5, 1-1-1 Higashi, Tsukuba, Ibaraki 305-8565, Japan.

*Corporate Research Center, R\&D Headquarters, Daicel Corporation, 1239, Shinzaike, Aboshi-ku, Himeji, Hyogo 671-1283, Japan.

$\S$ Advanced Materials Planning, R\&D Headquarters, Daicel Corporation, 2-19-1 Konan, Minato-ku, Tokyo 108-8230, Japan.

* To whom correspondence should be addressed. E-mail: e-miyako@aist.go.jp 
Supporting Figure and Table:

(A)



(B)

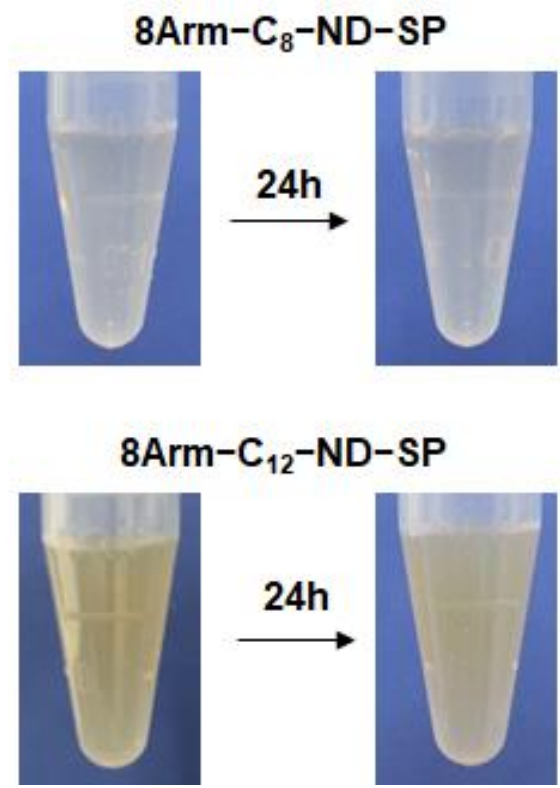

Supporting Figure S1. Water dispersibility of PTX-loaded 050GS-C12-ND-SP; ND and PTX concentrations are 0.3 and $0.1 \mathrm{mg} \mathrm{mL}^{-1}$, respectively; images of the synthesized products of 8 ArmC 8 -ND-SP and 8Arm-C $12-\mathrm{ND}-\mathrm{SP}$, which are prepared using $3 \mathrm{mg} \mathrm{mL}^{-1}$ of ND-SPs (Figure S1); list of abbreviations (Table S1). 
Table S1. List of abbreviations.

\begin{tabular}{|c|c|}
\hline Abbreviation & Meaning(s) \\
\hline 8Arm & Hexaglycerol octa(succinimidyloxyglutaryl)polyoxyethylene \\
\hline BODIPY & Boron dipyrromethene \\
\hline $\mathrm{C}_{8}$ & Sodium octanoate \\
\hline $\mathrm{C}_{12}$ & Sodium laurate \\
\hline $\mathrm{C}_{18}$ & Sodium oleate \\
\hline CCK & Cell counting kit \\
\hline $\mathrm{CV}$ & Crystal violet \\
\hline DLS & Dynamic light scattering \\
\hline DMEM & Dulbecco's modified Eagle's medium \\
\hline 050GS & $\alpha$-Succinimidyloxyglutaryl- $\omega$-methoxy polyoxyethylene \\
\hline MES & 2-(N-morpholino)ethanesulfonic acid \\
\hline ND & Nanodiamond \\
\hline ND-ori & Original nanodiamond \\
\hline NIR & Near infrared \\
\hline PEG & Polyethylene glycol \\
\hline PEGylation & Surface modification by a polyethylene glycol polymer \\
\hline PFOA & Perfluorooctanoic acid \\
\hline PTX & Paclitaxel \\
\hline SKOV3 & Ovarian cancer cells \\
\hline SP & Supraparticle \\
\hline TEM & Transmission electron microscopy \\
\hline TIG3 & Normal human diploid cells \\
\hline $\mathrm{U} 2 \mathrm{OS}$ & Bone osteosarcoma cells \\
\hline USFDA & United States of America Food and Drug Administration \\
\hline UV & Ultraviolet \\
\hline Vis & Visible \\
\hline WSC & $\begin{array}{l}\text { Water-soluble carbodiimide or 1-ethyl-3-(3- } \\
\text { dimethylaminopropyl)carbodiimide hydrochloride }\end{array}$ \\
\hline
\end{tabular}

